\title{
VALORES DE COLINESTERASAS EN
}

TRABAJADORAS ACTIVAS EMBARAZADAS, "MENSTRUANTES, USUARIAS DE ANTICONCEPTIVOS O MENOPÁUSICAS”

\author{
CHOLINESTERASE LEVELS IN ACTIVE WORKING, \\ PREGNANT, MENSTRUIATING, MENOPAUSAL, OR \\ CONTRACEPTIVE UISER WOMEN
}

Jaime Carmona-Fonseca, M.D. *

Recibido: diciembre 10/2002 - Revisado: abril 8/2003 - Aceptado: agosto 22/2003

\section{RESUMEN}

Problema: medir colinesterasas eritrocitaria (EC 3.1.1.7), plasmática (EC 3.1.1.8) y en sangre total, en mujeres trabajadoras activas clasificadas según su estado hormonal sexual (EHS).

Metodología: es un estudio descriptivo, prospectivo y transversal de valores de referencia de colinesterasas en población laboral del valle de Aburra y del cercano oriente antioqueño (Antioquia, Colombia), se hallaron 430 mujeres con información sobre EHS. Se midió la actividad enzimática en eritrocitos y plasma con técnicas electrométrica de Michel y colorimétrica EQM®, en plasma con Monotest ${ }^{\circledR}$ (cinética Boehringer Mannheim) y en sangre total con Lovibond $\AA$.

Resultados: en 430 de 437 mujeres se conoció «estado hormonal declarado» (embarazo, menstruación, otro). Al asociarlo con edad se construyó «estado hormonal deducido» (embarazo, menstruación, no embarazo-no menstruación, menopausia). Excluidas 21 anémicas quedaron 13 embarazadas, 47 menstruantes, 307 no embarazo-no menstruación,

Médico salubrista, epidemiólogo, ARP Seguro Social, Medellín. Profesor titular, facultad de Medicina, Universidad de Antioquía.
42 menopáusicas. Las colinesterasas eritrocitaria y en sangre total son estadísticamente iguales entre grupos, pero la plasmática es significativamente mayor en menopáusicas, luego en no embarazadas-no menstruantes, cae en menstruantes y es mínima en embarazadas. La diferencia la hacen mujeres menopaúsicas y embarazadas, que son distintas entre sí y con los otros dos grupos, pero los demás son similares entre ellos.

Conclusión: cualquiera que sea la técnica de medición de enzima plasmática, ésta cambia con el EHS: el menor nivel corresponde al embarazo y el mayor a la menopausia, que son los grupos femeninos con los niveles más altos y más bajos, respectivamente, de hormonas sexuales femeninas. En el intermedio aparecen las usuarias de terapia hormonal, las usuarias de anovulatorios y quienes no usan hormonas.

Palabras clave: colinesterasas, estado hormonal sexual, trabajadoras.

\section{SUMMARY}

Objective: to measure erythrocyte (EC 3.1.1.7), plasma (EC 3.1.1.8) and whole blood 
cholinesterases in active working women classified according to their sexual hormone status (SHS).

Methodology: in a cholinesterase reference values study (descriptive, prospective, cross sectional) carried out among the working population of valle de Aburra and Oriente Cercano Antioqueño (Antioquia Department, Colombia), a total of 430 women give information about SHS. The enzymatic activity was measured in plasma and erythrocytes using Michel's electrometric and EQM colorimetric techniques. Plasma values were also measured by Monotest ${ }^{\circledR}$ (Boehringer Mannheim kinetics) and whole blood values were measured by Lovibond ${ }^{\circledR}$, a colorimetric test.

Results: in 430 out of 437 women a "declared hormonal status" was known (pregnant, menstruating, other). Upon age association, a "deduced hormonal state" was established (pregnant, menstruating, non pregnant-non menstruating, menopause). During analyses, 21 women with anemia were excluded and the included (409 women) were distributed as follows: 13 pregnant, 47 menstruating , 307 non pregnantnon menstruating, 42 menopause. Erythrocyte and whole blood cholinesterase were statistically similar among the groups, but plasma values were significantly higher in menopause subjects, followed by non pregnant-no menstruating, lower in menstruating and very low in pregnant. The most significant difference was observed between the groups menopausic and pregnant. The two other groups evidenced similar values.

Conclusion: plasma concentration of the enzyme changes with SHS which ever test is applied. The lowest values were observed in pregnant and the highest in menopause women. These two groups coincide with the highest and the lowest female hormone load, between these two groups are the users of hormone therapy replacement, ovulation inhibitors users and those under no hormone treatment.

Keywords: cholinesterase, sexual hormone status, female workers.

\section{INTRODUCCIÓN}

Las enzimas que hidrolizan la acetilcolina y otros ésteres de la colina se llaman colinesterasas y son de dos clases: la acetilcolinesterasa o colinesterasa eritrocitaria (EC 3.1.1.7) y la butirilcolinesterasa, seudocolinesterasa o colinesterasa plasmática (EC 3.1.1.8). Hay un grupo de situaciones que modifican los niveles de colinesterasas, así:

1. Sexo en general: los promedios masculinos son estadísticamente superiores a los femeninos tanto de colinesterasa plasmática como eritrocitaria y en sangre total. ${ }^{1,2,3}$

2. Edad: es confusa la relación colinesterasas-edad, tanto en la eritrocitaria como en la plasmática y la total; ${ }^{4-9}$ en resumen, unos encuentran diferencias en función de la edad pero otros no las hallan.

3. Gestación: embarazo, aborto, puerperio y enfermedad trofoblástica

Durante la gestación se modifica la actividad colinesterásica:

a) la enzima eritrocitaria se eleva significativamente con respecto al estado de no embarazo, ${ }^{10-14}$ pero no cambia durante las semanas 14-20 de la gestación; ${ }^{15}$

b) la enzima plasmática se reduce en el estado gestante $^{13,14,16}$ y también se informa que una minoría de mujeres no presenta cambio. ${ }^{16} \mathrm{La}$ colinesterasa plasmática muestra una caída brusca en el primer trimestre, en un orden de magnitud del 20\% de la actividad, con recuperación leve en el tercer trimestre y niveles más bajos del segundo al séptimo día postparto. La recuperación total (nivel de no embarazada) ocurre de 3 a 6 semanas después del parto. ${ }^{13,16,17,18,19}$

Se han identificado tres patrones de comportamiento temporal de la enzima plasmática asociados al embarazo, así: reducción posconcepción con no retorno a nivel preconcepción (disminución continua) en $46 \%$ de 44 gestaciones evalua- 
das; reducción posconcepción con retorno parcial o total a nivel preconcepción (disminución transitoria) en $43 \%$ y no reducción o aumento durante gestación (no cambio) en 11\%.

Los autores no encontraron asociación del patrón de cambio enzimático gestacional con el sexo del recién nacido, la paridad, el antecedente de fumar o la historia de ingesta de alcohol, pero si con la edad materna: el patrón de reducción continua ocurrió predominantemente en las mujeres más jóvenes, el patrón de reducción transitoria en las mujeres de edad intermedia y el patrón de no cambio en las mujeres de más edad. Con base en lo anterior concluyen que la variación gestacional de la enzima plasmática puede estar influida por la madurez física materna. ${ }^{20}$

En el aborto espontáneo ocurre una intensa reducción de la actividad enzimática plasmática ${ }^{21}$ e igual sucede en la enfermedad trofoblástica. ${ }^{22}$

4. Hemoglobina, hematocrito, edad de los eritrocitos

La acetilcolinesterasa disminuye en las células eritrocitarias de mayor edad. En los niños, la máxima actividad se presenta en el período correspondiente al 10\% inicial de la vida eritrocitaria y disminuye en el $70 \%$ final. En los adultos el incremento se produce en el primer 25\% de la vida del eritrocito y el descenso en el $50 \%$ final. ${ }^{23}$ También se sabe que en los eritrocitos senescentes hay reducción de la enzima eritrocitaria. ${ }^{24}$

La medida de la hemoglobina empleada en uno de los métodos de laboratorio para colinesterasas eritrocitaria (método de EQM@) se usa para corregir las variaciones de la enzima procedente de dos fuentes: la primera se refiere al hematocrito, el cual puede variar en más de un 50\% de una persona a otra, y la segunda fuente está en el error del muestreo de sangre. ${ }^{2}$

5. Enfermedades y estados clínicos

Diversas enfermedades y condiciones mórbidas alteran los niveles de la actividad colinesterásica plasmática, ya sea disminuyéndolos ${ }^{17,25}$ o elevándolos. ${ }^{17,26,27}$ Desnutrición, anemia crónica, hepatopatías, infecciones, anovulatorios orales, estrógenos y corticosteroides están entre las causas de reducción de la enzima plasmática, mientras que estados de ansiedad, obesidad, alcoholismo e hipertensión figuran entre las que ocasionan incremento. También se conoce de situaciones que modifican la colinesterasa eritrocitaria, como el embarazo ${ }^{10-19}$ y la desnutrición..$^{28,29}$

\section{Condición genética}

Un 3\% de los caucásicos y menos del 1\% de los negros presentan normalmente niveles bajos de actividad colinesterásica plasmática. ${ }^{30,31,32}$ Se sabe de la existencia de deficiencia hereditaria de la colinesterasa eritrocitaria. ${ }^{33}$ Se ha informado de cierta relación del factor $\mathrm{Rh}$ (determinado en forma genética) con la colinesterasa plasmática entre mujeres embarazadas, asociación no encontrada en no grávidas ni en hombres. ${ }^{20}$ Otros autores no han hallado tal asociación. ${ }^{34}$

Existe un polimorfismo de supresión/inserción del gen de la colinesterasa y hay una asociación entre los genotipos de este polimorfismo y los niveles séricos de la enzima; la fuerza de esta influencia varia en poblaciones de diferente origen étnico. ${ }^{35}$ Además de las variaciones normales de índole genética como las antes referidas, los niveles de la enzima pueden verse afectados por variantes genéticas disfuncionales, por exposición a inhibidores enzimáticos y por biosíntesis reducida. ${ }^{36,37,38}$

\section{Factores fisioquímicos}

Ando y colaboradores describieron en 1984, una interesante relación lineal entre la actividad de la colinesterasa plasmática $(\mathrm{CP})$ y varios factores fisioquímicos (edad, transaminasa glutámicopirúvica, hemoglobina, colesterol total). ${ }^{39}$ Las estimaciones de la actividad enzimática obtenidas con este modelo concuerdan con la actividad medida y los cambios significativos de actividad pueden ser 
atribuidos a efectos tóxicos de plaguicidas o a determinada anormalidad de la función hepática.

El presente informe da cuenta de los valores de colinesterasas eritrocitaria (EC 3.1.1.7) y plasmática (EC 3.1.1.8) en varios grupos de mujeres, hallados en un estudio representativo de la población laboral afiliada al Seguro Social y vinculada a empresas localizadas en el valle de Aburrá o en el cercano oriente antioqueño (Antioquia, Colombia).

Los grupos corresponden a 410 mujeres sin anemia (cianometahemoglobina de 12 o más g/ $\mathrm{dL}$ ), bien sea embarazadas, en fase menstrual o sin menstruación ni embarazo y en edad reproductiva. También se hace una discriminación según estado reproductivo: mujeres en menopausia, usuarias de anovulatorios orales y de terapia hormonal sexual.

\section{MATERIALES Y MÉTODOS}

\section{Diseño de la muestra poblacional}

Los detalles del diseño y cálculo muestrales se informaron antes. ${ }^{3}$ El estudio descriptivo, prospectivo y transversal se diseñó para obtener valores de referencia de colinesterasas en población laboral activa, no expuesta a plaguicidas inhibidores de colinesterasa (PIC). Al final se incluyeron personas de 18 a 75 años, pero los estratos con diseño estadístico y epidemiológico adecuado se refieren únicamente a los menores de 50 años: 18-29, 30-39, 40-49 años; los otros dos grupos (50-59 y 60-75) se incluyeron sólo para fines comparativos y exploratorios.

Las personas estudiadas fueron tomadas al azar entre quienes aceptaron participar en el estudio y llenaron los requisitos de inclusión. Los participantes dijeron sentirse bien y estaban trabajando en el momento de ingreso al estudio.

\section{Criterios de inclusión}

Mediante una entrevista personal a cada trabajador, se investigó la presencia de enfermedades que alteran los niveles de colinesterasas y quien tuvo alguna de ellas se excluyó del estudio.

Los estados fisiológicos como embarazo y menstruación, que modifican los valores de actividad colinesterásica, no fueron causa de exclusión. Tampoco lo fue la ingestión de drogas si el trabajador decía sentirse bien, pero quienes dijeron tomar medicamentos y afirmaron no estar con buena salud quedaron por fuera del estudio. Estos dos grupos se dejaron con el fin de compararlos con aquel otro conformado por quienes ni estaban enfermos, ni en embarazo, ni menstruaban, ni tomaban drogas.

Todos los trabajadores incluidos laboraban en empresas donde ni por rutina ni ocasionalmente se usan PIC. Además se indagó a los trabajadores sobre el uso extralaboral de tales sustancias en el último mes, excluyendo a quienes manifestaron haberlos usado en actividades como aplicación domiciliaria contra insectos, plagas de jardín u otras similares.

\section{Métodos y técnicas de laboratorio}

Las colinesterasas se midieron por seis técnicas diferentes: electrométrica de Michel para eritrocitos y plasma, colorimétrica con equipo EQM® para eritrocitos y plasma, basada en el principio de Ellman y modificado por Magnotti (Cincinnati, Ohio, USA), cinética con reactivos Boehringer Mannheim para plasma (Monotest $\left.{ }^{\circledR}\right)$ y colorimétrica para sangre total (Tintométrica de Lovibond $\left.{ }^{\circledR)}\right)^{3}$

La hemoglobina se midió por dos técnicas diferentes: una mide la cianometahemoglobina y la otra la oxihemoglobina. Esta última hace la medición como un paso intermedio en la determinación de los niveles de colinesterasa eritrocitaria, la cual debe ser ajustada en función de los valores de oxihemoglobina. ${ }^{3}$ Las unidades de medición de las técnicas son: deltas de $\mathrm{pH} /$ hora para Michel en eritrocitos y plasma, $\mathrm{U} / \mathrm{g}$ de oxihemoglobina en EQM eritrocitario, U/mL en EQM plasmático, U/ 
L en Monotest (estas unidades son diferentes a las de EQM) y porcentaje en Lovibond.

\section{Análisis estadístico}

Para el análisis estadístico se usaron los programas SGPlus versión 7,1 y EpiInfo versión 6,04. Siempre se aplicó un nivel de significación menor de 5\% ( $<<0,05)$. En el análisis de varianza (anova): a) las sumas de cuadrados se obtuvieron por el procedimiento de tipo III para tales sumas y todas las razones $\mathrm{F}$ están basadas en el error residual de mínimos cuadrados; b) los gráficos para representar las diferencias entre las medias y los intervalos de confianza del 95\% (IC95\%) se hicieron siempre por la prueba HSD de Tukey al 95\%; c) en el análisis de rango múltiple (ARM) siempre se usó el método de Newman-Keuls al 95\%.

\section{RESULTADOS}

Se estudiaron 827 personas: 415 fueron del Aburrá y 412 del Oriente. Un total de 437 eran mujeres, 222 de Aburrá y 215 de Oriente. De las 437 mujeres, en 430 se captó el dato sobre su "estado hormonal declarado", entendiendo por ello su informe sobre la presencia de menstruación en el día de la encuesta o de embarazo. Quienes dijeron que no estaban menstruando ese día ni estaban en embarazo, se clasifican como "no embarazo y no menstruación”. Se hallaron en total 14 embarazadas, 53 menstruantes y las restantes 363 mujeres manifestaron no estar en embarazo y no tener menstruación en ese momento (entre ellas es posible que existan algunas embarazadas, que aún no han descubierto su situación, pero deben ser muy pocas).

$\mathrm{Al}$ analizar los datos, se tomaron las $363 \mathrm{mu}-$ jeres en la categoría "no embarazo-no menstruación" de la variable "estado hormonal declarado" y, considerando su edad (menos de 50 años o 50 y más años), se clasificaron como no menopáusicas y menopáusicas, respectivamente, quedando con menopausia 43 mujeres (una de ellas tiene 50 años y dijo tener menstruación, lo que demuestra que el supuesto de menopausia a partir de los 50 años es bastante adecuado pero no absoluto).

Con esta clasificación del "estado hormonal deducido por el investigador" resultan cuatro grupos de mujeres: $\sin$ menstruación y $\sin$ embarazo $(\mathrm{n}=$ 321), con menstruación ese día $(n=52$, en lugar de las 53 que "declararon”), con embarazo $(\mathrm{n}=14$, igual a lo "declarado") y menopausia $(n=43)$. Los tres primeros grupos corresponden a un "estado hormonal sexual activo" (excepto 3 mujeres, las demás están en edad reproductiva), mientras el cuarto es "estado sexual inactivo" (tabla 1 , parte $A)$.

Si se separan las mujeres con cianometahemoglobina menor de $12 \mathrm{~g} / \mathrm{dL}$, los grupos se reducen a 13 embarazadas, 47 menstruantes, 307 sin ninguno de los dos estados anteriores y 42 con menopausia, para un total de 409 mujeres.

Se evaluó el comportamiento de las colinesterasas en las 409 mujeres sin anemia repartidas en los cuatro grupos y no tienen diferencia estadísticamente significativa (d.e.s.) en sus colinesterasas eritrocitarias ni de sangre total pero si tienen d.e.s. en las enzimas plasmáticas (tabla 1, parte B).

Los valores de colinesterasa plasmática, por cualquier técnica de laboratorio, son mayores en las menopáusicas, luego en no embarazadas-no menstruantes, caen en las menstruantes y se reducen mucho más en las embarazadas. Los grupos que se comportan diferente son los de menopáusicas y embarazadas, pues la d.e.s. siempre están entre ellos, entre si y con cada uno de los otros dos (menstruantes y no menstruantes-no embarazadas), pero nunca hay d.e.s. entre menstruantes y no embarazadas-no menstruantes. Dentro del grupo "no menstruación-no embarazo" los niveles de enzima plasmática son estadísticamente diferentes según la edad, pero 


\section{Tabla 1. Estado hormonal sexual y colinesterasas plasmáticas en mujeres sin anemia}

Parte A. Distribución de las 430 mujeres según «estado hormonal sexual» y edad

\begin{tabular}{|c|c|c|c|c|c|c|c|c|}
\hline Edad & $18-29$ & $30-39$ & $40-49$ & $50-59$ & $60-75$ & total & sin anemia & $\begin{array}{c}\text { IC95\% } \\
\text { edad }\end{array}$ \\
\hline $\begin{array}{l}\text { no-M, } \\
\text { no- } E^{(1)}\end{array}$ & 155 & 103 & 60 & 3 & 0 & 321 & 307 & $30-32$ \\
\hline Embarazo & 10 & 3 & 1 & 0 & 0 & 14 & 13 & $24-32$ \\
\hline Menstruo & 32 & 14 & 6 & 0 & 0 & 52 & 47 & $26-30$ \\
\hline Menopausia & 0 & 0 & 0 & 25 & 18 & 43 & 42 & $56-60$ \\
\hline Total & 197 & 120 & 67 & 28 & 18 & 430 & & $31-34$ \\
\hline Total sin & 187 & 112 & 65 & 28 & 17 & & 409 & $33-34$ \\
\hline & \multicolumn{3}{|c|}{ Edad reproductiva } & \multicolumn{3}{|c|}{ No edad reproductiva } & & \\
\hline & \multicolumn{3}{|c|}{ Ciclo menstrual activo } & \multicolumn{3}{|c|}{ Menopausia } & & \\
\hline
\end{tabular}

${ }^{(1)}$ no-M,no-E no menstruación y no embarazo; excepto 3 mujeres, las demás están en edad reproductiva

la diferencia existe entre las menores de 50 años y las de 50 y más años, siendo iguales los valores entre los tres grupos etáreos con "ciclo menstrual activo" y entre los dos grupos en "menopausia". Se anota que por ninguna de las dos técnicas de medición, la enzima eritrocitaria muestra diferencia según el estado hormonal.

Se hallaron 8 mujeres que usaban anovulatorios orales (edad: 18-39 años) y otras 8 que recibían terapia hormonal con estrógenos orales (estrógenos naturales conjugados equinos, valerianato de estradiol) o con progestágeno oral o de depósito (acetato de medroxiprogesterona).

Se excluyeron las mujeres con anemia (cianometahemoglobina menor de $12 \mathrm{~g} / \mathrm{d}$; una usaba anovulatorios) y, entre las mujeres con "no embarazo-no menstruación”, se compararon los promedios de colinesterasa plasmática de las 6 usuarias de anovulatorios, las 3 usuarias de terapia hormonal y las 298 no usuarias de ninguno de los dos tratamientos; los promedios son menores en las usuarias de terapia hormonal, luego están las que usan anovulatorios y los más altos niveles están en quienes no emplean hormonas (ni como anticonceptivos ni como terapia), pero la diferencia es insignificante (tabla 2).

\section{DISCUSIÓN}

Nuestros datos difieren de lo hallado por González-Horta y asociados, quienes informaron que la actividad de colinesterasa eritrocitaria (acetilcolinesterasa) en mujeres en el tercer trimestre de gestación y en trabajo de parto fue mayor que en aquellas en tercer trimestre de la gestación y que los más bajos niveles estuvieron en mujeres sanas no embarazadas ${ }^{10}$. Nosotros no hallamos diferencia en ninguno de los cuatro grupos comparados (gestantes, menstruantes, no embarazadas-no menstruantes, en menopausia). Existen informes que afirman que la enzima no cambia durante las semanas 14-20 de la gestación. ${ }^{15}$

La mayoría de informes señalan que la colinesterasa plasmática es menor en mujeres embarazadas, ${ }^{13,14,16,18,19,39}$ comparadas con otras de la misma edad no grávidas. Nuestros hallazgos coinciden con este informe y las diferencias halladas entre gestantes y no gestantes son estadísticamente significativas, pero no los otros dos grupos entre si 


\section{Tabla 1. Estado hormonal sexual y colinesterasas} plasmáticas en mujeres sin anemia

Parte B. Colinesterasas plasmáticas según «estado hormonal sexual» y técnica de medición enzimática en mujeres sin anemia ${ }^{(2)}$

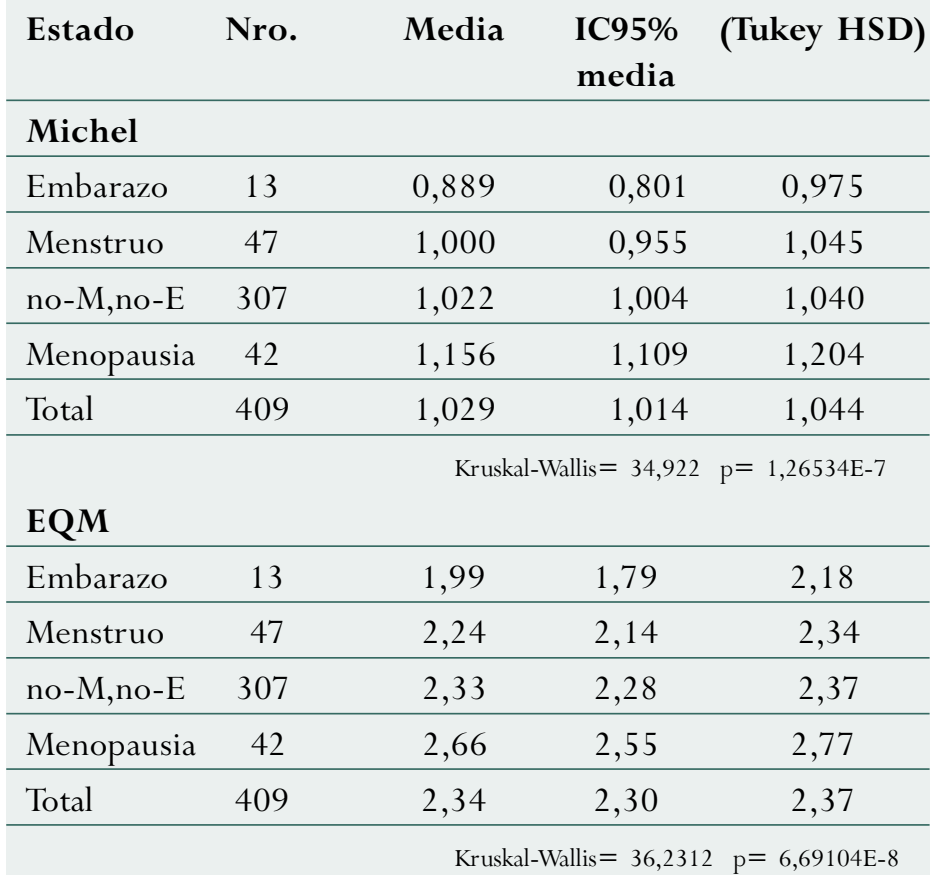

Monotest

\begin{tabular}{|c|c|c|c|c|}
\hline Embarazo & 13 & 4195 & 3665 & 4724 \\
\hline Menstruo & 47 & 4889 & 4610 & 5168 \\
\hline no-M,no-E & 307 & 5094 & 4985 & 5203 \\
\hline Menopausia & 42 & 5941 & 5647 & 6236 \\
\hline Total & 409 & 5129 & 5034 & 5223 \\
\hline
\end{tabular}

(2) En cualquier técnica de medición, los promedios tienen siempre este orden: menopausia $>$ no menstruación y no embarazo $>$ menstruo $>$ embarazo. Los grupos que marcan diferencia son menopausia y embarazo que difieren entre si y con cada uno de los otros dos, que nunca difieren entre ellos.

(en fase menstrual y menopausia). Muy interesante es el hallazgo de la relación entre el nivel de colinesterasa plasmática (butirilcolinesterasa) y el estado hormonal: el nivel mínimo está en la gestación, aumenta en fase menstrual, sube más en no embarazadas-no menstruantes y llega al máximo en la menopausia. La influencia hormonal sexual sobre el nivel de colinesterasa plasmática es evidente y significativa y se corrobora cuando dentro del grupo «no menstruación-no embarazo» se comprueba que la enzima plasmática difiere estadísticamente según la edad y que tal diferencia existe únicamente entre las menores de 50 años y las de 50 y más años, siendo iguales los valores entre los tres grupos etáreos con «ciclo menstrual activo» y entre los dos grupos en «menopausia».

En ese grupo (no menstruación-no embarazo), la correlación entre edad y nivel de enzima plasmática es casi nula, con cualquiera de las tres técnicas de medición (siempre el coeficiente $r$ es menor de 0,16). Esto sugiere que la edad afecta los valores de colinesterasa plasmática mediante la influencia que ejercen las hormonas sexuales.

González-Horta y colaboradores han informado que el nivel de actividad plasmática de las gestantes no se asocia con tabaquismo, índice de masa corporal, uso de insecticidas en el hogar o niveles de hemoglobina. ${ }^{10}$ Nosotros también encontramos que, entre las gestantes, la actividad de la enzima plasmática no cambia según la presencia de anemia (definida como menos de 12 g/dL de cianometahemoglobina).

Trece de las 14 embarazadas presentaron niveles de la enzima por debajo del límite inferior que tenían las no embarazadas-no menstruantes; la excepción fue una mujer de 23 años; la edad de estas mujeres tiene una mediana de 26 años y un promedio de 27 años, el mínimo fue 20 y el máximo 40; no se tiene informe de la edad de la gestación. Esto se señala a raíz del interesante hallazgo de Evans y colaboradores, quienes encontraron asociación del patrón de cambio enzimático gestacional con la edad materna: el modelo de reducción continua ocurrió especialmente en mujeres más jóvenes, la reducción transitoria en mujeres de edad intermedia y el patrón de 
Tabla 2. Colinesterasa plasmática en mujeres sin anemia usuarias de

hormonas sexuales (anovulatorios [An.] o terapia hormonal sexual [THS]), según «estado hormonal sexual» y técnica de medición

A. Tamaño de los grupos según Estado hormonal sexual

\begin{tabular}{lcccc}
\hline Estado hormonal & No usa & An. & THS & Total \\
\hline No menstruo-no embarazo & 298 & 6 & 3 & 307 \\
\hline Menstruación & 45 & 1 & 1 & 47 \\
\hline Menopausia & 38 & 0 & 4 & 42 \\
\hline Total & 381 & 7 & 8 & 39 \\
\hline
\end{tabular}

B. Promedios de colinesterasa plasmática en el grupo «no menstruación-no embarazo», según uso de hormonas sexuales femeninas como anovulatorios (A) o terapia de reemplazo (TR) ${ }^{(1)}$

\begin{tabular}{|c|c|c|c|}
\hline \multirow[t]{2}{*}{ Estado hormonal } & A & TR & No usa \\
\hline & $n=6$ & $\mathrm{n}=3$ & $\mathrm{n}=298$ \\
\hline Michel deltas $\mathrm{pH} /$ hora & 0,983 & 0,933 & 1,021 \\
\hline EQM U/mL & 2,27 & 2,37 & 2,33 \\
\hline Monotest $\mathrm{U} / \mathrm{mL}$ & 5036 & 4804 & 5098 \\
\hline \multicolumn{4}{|c|}{$\begin{array}{l}\text { (1) no hay d.e.s. entre los } 3 \text { grupos (no usa hormonas, anovulatorios, terapia } \\
\text { hormonal sexual), en ninguna de las técnicas. } \\
\text { C. Promedios de colinesterasa plasmática en menopáusicas, según uso } \\
\text { de terapia hormonal sexual }\end{array}$} \\
\hline \multirow[t]{2}{*}{ Estado hormonal } & No usa & \multicolumn{2}{|c|}{ Si usa } \\
\hline & $\mathrm{n}=38$ & \multicolumn{2}{|c|}{$\mathrm{n}=4$} \\
\hline Michel deltas $\mathrm{pH} /$ hora & 1,150 & \multicolumn{2}{|c|}{1,175} \\
\hline EQM U/mL & 2,62 & \multicolumn{2}{|c|}{$3,03 \mathrm{p}<0,05^{(1)}$} \\
\hline Monotest $\mathrm{U} / \mathrm{mL}$ & 5832 & \multicolumn{2}{|c|}{6974} \\
\hline
\end{tabular}

(1) $\mathrm{K}-\mathrm{W}=4,321 ; \mathrm{gl}=1 ; \mathrm{p}=0,037649$

no cambio en las mujeres de más edad, concluyendo que la variación gestacional de la enzima plasmática puede estar influida por la madurez física materna. ${ }^{20}$

Comparadas con mujeres de edad similar, sin anemia, sin menstruación ni embarazo, nosotros encontramos los niveles más altos de enzima plasmática en las usuarias de terapia hormonal, seguidas de las usuarias de anovulatorios orales, aunque la diferencia no es significativa, siendo posible que el escaso número de mujeres en dos de los grupos responda por parte de esta ausencia de diferencia.
De todas maneras, coincidimos con den Blaauwen y asociados, quienes informaron que la colinesterasa plasmática es menor en mujeres que toman anticonceptivos orales, comparadas con otras de la misma edad. ${ }^{40}$ De nuevo, es muy claro que las hormonas sexuales femeninas, como anovulatorios y, todavía más, como terapia de reemplazo, reducen los niveles de colinesterasa plasmática.

Con la prueba Monotest ${ }^{\circledR}$, para la enzima en plasma, den Blaauwen y su grupo informan valores de referencia iguales para niños y niñas (1-15 años), hombres adultos (16-94 años) y mujeres adultas mayores (40-99 años) no embarazadas ni usuarias de anticonceptivos hormonales, con valores de 3500 a 8500 U/L (marca de clase: 6000); para mujeres adultas jóvenes (16-39 años) no embarazadas ni usuarias de anticonceptivos indican 2800 a $7400 \mathrm{U} / \mathrm{L}$ (marca de clase: 5100) y para mujeres adultas (18-41 años) embarazadas o que toman anticonceptivos señalan 2400 a 6000 U/L (marca de clase: 4200$){ }^{40}$

Nosotros obtuvimos niveles similares a los estos autores en los dos primeros grupos: 5822 en hombres (18-75 años) y 5897 en mujeres adultas de 40 y más años, por un lado, y, por otra parte, 5062 (IC95\%: 4952 a 5171) en mujeres jóvenes (18-40 años) no embarazadas, no usuarias de anticonceptivos hormonales y sin menstruación activa. 
La única diferencia aparece en el grupo de gestantes o usuarias de anticonceptivos hormonales, para quienes los autores citados entregan valores iguales, lo que es distinto de nuestros hallazgos, que señalan valores iguales a los de den Blaauwen y asociados en las embarazadas (4195 frente a $4200 \mathrm{U} / \mathrm{L}$ ) pero con diferencia entre esas mujeres y las que toman anticonceptivos, quienes tienen $5036 \mathrm{U} / \mathrm{L}$, valores que son un poco mayores pero estadísticamente similares a los de mujeres en menopausia que usan terapia con hormonas sexuales, quienes poseen $4804 \mathrm{U} / \mathrm{L}$.

En resumen, la concentración de hormonas sexuales femeninas se asocia con la actividad de colinesterasa plasmática en forma inversa, pues la mayor reducción corresponde al embarazo y la menor a la menopausia, que son los grupos con los niveles de hormonas más altos y más bajos, respectivamente. Esta relación se encuentra cualquiera que sea la técnica de medición enzimática.

En el intermedio de esos dos extremos aparecen las usuarias de terapia hormonal, las usuarias de anovulatorios y quienes no usan terapia con hormonas sexuales, en un orden que varía según la técnica para medir la colinesterasa, pero que deja a las no usuarias de hormonas con niveles de butirilcolinesterasa por encima del que tienen las usuarias de anticonceptivos o de terapia hormonal.

La única situación conflictiva en este enfoque la producen las mujeres en menopausia según usen o no terapia hormonal, pues el nivel enzimático es mayor entre quienes la emplean, que es contrario a lo esperado según el análisis que se hace. De todas formas habrá que estudiar más el asunto, ya que la única diferencia significativa se halló con la técnica de EQM.

\section{AGRADECIMIENTOS}

- Al médico salubrista Samuel Henao H, a las químicas Flor María Zapata L y Rocío Garcés M, a la médica de salud ocupacional María Isa- bel Gallego P, quienes, junto con el autor, crearon el proyecto de investigación. Las doctoras Zapata y Garcés realizaron las mediciones químicas (Michel, EQM). Por diversas razones, todos estos profesionales ya no están vinculados al Seguro Social.

- Al Laboratorio de Toxicología de la Facultad de Medicina en la Universidad de Antioquia, por la realización de las mediciones con Monotest, y al Laboratorio Clínico Hematológico Ltda. (Medellín), por las mediciones de cianometahemoglobina.

- A Gabriel Agudelo V, estadístico y bioestadístico, profesor titular, Departamento de Matemáticas de la Universidad de Antioquia, por su asesoría.

- A la Facultad Nacional de Salud Pública, Universidad de Antioquia, por el apoyo administrativo al proyecto.

- Al Fondo de Promoción de la Salud Industrial del Seguro Social (Colombia), a la Administradora de Riesgos Profesionales Seguro Social, seccional Antioquia, y la Universidad de Antioquia, por la cofinanciación del proyecto.

\section{REFERENCIAS}

1. Rider JA, Hodges JL Jr, Swader J, Wiggins AD. Plasma and cell cholinesterase in 800 "healthy" blood donors. J Lab Clin Med. 1957; 50: 376-383.

2. EQM Research Inc. (Cincinnati, Ohio, USA). Cholinesterase kit for the field determination of pesticide exposure. Instruction manual. Cincinnati (Ohio): EQM, 1994.

3. Carmona-Fonseca J, Henao S, Garcés R. Valores de referencia de actividad colinesterásica sanguínea en población laboral activa no expuesta a plaguicidas inhibidores de colinesterasa. Rev Fac Nal Sal Públ (Medellín) 2000; 18 (2): 55-72.

4. Henao S, Corey G. Plaguicidas inhibidores de las colinesterasas. Serie Vigilancia 11. Centro Panamericano de Ecología Humana y Salud. Metepec, México: ECO, OPS, OMS, 1991. 
5. Abou-Hatab K, O’Mahony MS, Patel S, Woodhouse K. Relationship between age and plasma esterases. Age Ageing 2001; 30 (1): 41-45.

6. Henao S, Zapata FM, Restrepo MP, Marín LE, Ramírez $\mathrm{H}$, Corrales R et al. Actividad colinesterásica en menores trabajadores. Antioquia (Colombia), 19891990. Medellín: Instituto de Seguros Sociales y Universidad de Antioquia, 1990.

7. Karlsen RL, Sterri S, Lyngaas S, Fonnum F. Reference values for erythrocite acetylcholinesterases and plasma cholinesterase activities in children, implications for organophosphate intoxication. Scand J Clin Lab Invest. 1981; 41 (3): 301-302.

8. García-López JA, Monteoliva M. Physiological changes in human erythrocyte cholinesterase as measured with the "pH-stat". Clin Chem. 1988; 34 (19): 2133 2135.

9. Chan L. Blood cholinesterase levels in the elderly and newborn. Malays J Pathol 1995 Dec;17(2):87-9

10. González-Horta MC, Casillas-Ituarte N, ErivesQuezada G, Reza-López S, Sanín-Aguirre LH, Levario-Carrillo M. Actividad de la acetilcolinesterasa durante el embarazo y en el recién nacido. Ginecol Obstet Méx. 2000; 68 (6): 231-235.

11. Rumenjak V. Distribution of human erythrocytes cholinesterase according to age, sex and pregnancy. Acta Med Croatica 1998; 52 (3): 187-189.

12. Brock DJ, Barron L. Prospective prenatal screening for fetal abnormalities using a quantitative immunoassay for cholinesterase. J Med Genet 1988; 25 (9): 606-608.

13. de Peyster A, Willis WO, Liebhaber M. Cholinesterase activity in pregnant women and newborns. J Toxicol Clin Toxicol 1994; 32 (6): 683-696.

14. Venkataram BV, Iyer GY, Narayanan R, Joseph T. Erythrocyte and plasma cholinesterase activity in normal pregnancy. Indian J Phisiol Pharmacol 1990; 34 (1): 26-28.

15. Brennand DM, Jehanli AM, Wood PJ, Smith JL. Raised levels of maternal serum secretory acetylcholinesterase may be indicative of fetal neural tube defects in early pregnancy. Acta Obst Gynecol Scand 1998; 77 (1): 8-13.

16. Evans RT, Wroe J M. Plasma cholinesterase changes during pregnancy. Their interpretation as a cause of suxamethonium-induced apnoea. Anaesthesia 1980; 35 (7): 651-654.
17. Whittaker M. Cholinesterase. New York, Karger Basel. 1986. Monographs in Human Genetics, $N^{\circ}$ 11.

18. Howard JK. Plasma cholinesterase activity in early pregnancy. Arch Environ Health 1978; 33 (5): 277-279.

19. Robson N, Robertson I, Whittaker M. Plasma cholinesterase changes during the puerperium. Anaesthesia 1986; 41 (3): 243-249.

20. Evans RT, O'Callaghan J, Norman A. A longitudinal study of cholimnesterase changes in pregnancy. Clin Chem 1988; 34 (11): 2249-2252.

21. Whittaker M, Crawford JS, Lewis M. Some observations of levels of plasma cholinesterase activity within an obstetric population. Anaesthesia 1988; 43 (1): 42-45.

22. Davies JM, Carmichael D, Dymond C. Plasma cholinesterase and trophoblastic disease. Gestational trophoblastic disease and reduced activity of plasma cholinesterase. Anaesthesia 1983; 38 (11): 1071 1074

23. Galbraith DA. Human erythrocyte acetylcholinesterase in relation to cell age. Biomedical J. 1981; 195: 221-228.

24. Kamada T, McMillan DE, Yamashita T, Otsuji S. Lowred membrane fluidity of younger erythrocytes in diabetes. Diabetes Res Clin Pract 1992; 16 (1): $1-6$.

25. Hayes W Jr. Pesticides studies in man. Baltimore, Maryland, USA, Williams and Wilkins, 1982.

26. Reuter W, Geus P. [Behavior of cholinesterase activity in lipid metabolism disorders]. Z Gesamte Inn Med 1987 Jul 1;42(13):371. Resumen consultado el 1 noviembre 2002 en: www.ncbi.nlm.nih.gov PMID 3630286 .

27. Kaloyanova F. Toxicity of selected groups of pesticides: organophosfates. In: World Health Organization, Regional Office for Europe. Toxicology of pesticides. Copenhagen, Denmark: WHO, 1982: 133-144. Health Aspects of Chemical Safety; Interim Document $\mathrm{N}^{\circ} 9$.

28. Jain AK, Hussain S, Ahuja S. Undernutrition in children. Effect on venocuronium indiced neuromuscular blockade. Anaesth Intensive Care 1999; 27 (4): 381-384.

29. Camarero-González E, Muñoz V, Iglesias M, Fernández JA, Cabezas J. Desnutrición proteico-ca- 
lórica: sus efectos sobre cuatro parámetros metabólicos. Nutr Hosp 1995; 10 (3): 158-160.

30. Garry PJ. A manual and automated procedure for measuring serum cholinesterase activity and identifying enzyme variants. Clin Chem Acta 1971; 17 (3): 192-198.

31. Coye MJ, Lowe JA, May KJ. Biological monitoring of agricultural workers exposed to pesticides: J Occupational Med 1986; 28 (8): 619-627 (part I); 628-638 (part II).

32. Kalow WGK. A method for determination of atypical forms of human serum cholinesterase: determination of dibucaine number. Canadian J Biochemical Physiology 1957; 35: 339.

33. Shinohara K, Tanaka KR. Hereditary deficiency of erythrocyte acetylcholinesterase. Am J Hematol 1979; 7 (4): 313-321.

34. Primo-Parmo SL, Chautard-Freire-Maia EA. Absense of linkage between the serum cholinesterase (CHE1) and rhesus (RH) loci. Hum Genet. 1982; 60 (3): 284-286.

35. Ruprecht B, Schurmann M, Ziegenhagen MW, vom Bauer E, Meir D, Schlaak M et al. Corrected normal values for serum ACE by genotyping the deletion/ insertion-polymorphism of the ACE gene. Pneumologie 2001; 55 (7); 326-332. Resumen consultado el 31 octubre 2002 en www.ncbi. nlm.nih.gov. PMID: 11481579.
36. Morton F. Detection of cholinesterase inhibition. The significance of cholinesterase measurements. Ann Clin Lab Sci. 1988; 18 (5); 345-452.

37. Gerreiro, JF, Santos, SEB, Black, FL. Frequencies of the atypical and $\mathrm{C} 5$ variants of serum cholinesterase in Wayana - Apalai Indians. Rev Bras Genet. 1985; 8 (1): 123-129.

38. Rosalki SB. Genetic influences on diagnostic enzymes in plasma. Enzyme 1988; 39 (2): 95-109.

39. Ando M, Hirosaki S, Tamura K, Taya T. Multiple regression analysis of the cholinesterase activity with certain physiochemical factors. Environ Res. 1984; 33 (1): 96-105.

40. den Blaauwen DH, Poppe WA, Tritschler W. Cholinesterase (EC 3.1.1.8) with butyrylthiocholineiodide as substrate: references depending on age and sex with special reference to hormonal effects and pregnancy. J Clin Chem Clin Biochem 1983; 21: 381-388. Rresumen consultado el 1 noviembre 2002 en: www.ncbi.nlm.nih.gov. PMID 6886638. Citado por Boehringer Mannheim en el folleto instructivo sobre Monotest ${ }^{\circledR}$ (Boehringer Mannheim). 